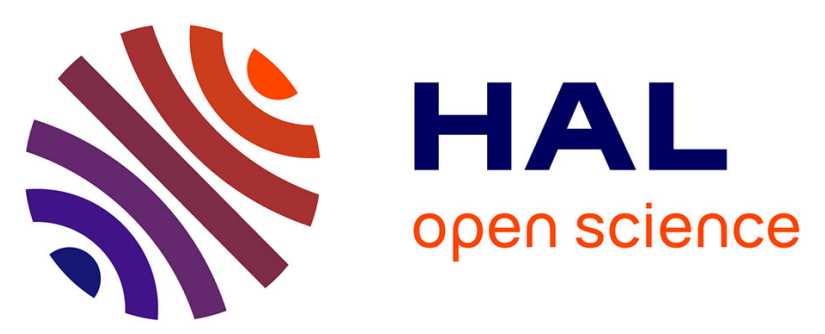

\title{
A LINEARITY PRESERVING CELL-CENTERED SCHEME FOR THE HETEROGENEOUS AND ANISOTROPIC DIFFUSION EQUATIONS ON GENERAL MESHES
}

Gao Zhiming, Wu Jiming

\section{To cite this version:}

Gao Zhiming, Wu Jiming. A LINEARITY PRESERVING CELL-CENTERED SCHEME FOR THE HETEROGENEOUS AND ANISOTROPIC DIFFUSION EQUATIONS ON GENERAL MESHES.

[Research Report] 2010. inria-00481112v1

HAL Id: inria-00481112

https://hal.inria.fr/inria-00481112v1

Submitted on 7 May 2010 (v1), last revised 16 Nov 2013 (v2)

HAL is a multi-disciplinary open access archive for the deposit and dissemination of scientific research documents, whether they are published or not. The documents may come from teaching and research institutions in France or abroad, or from public or private research centers.
L'archive ouverte pluridisciplinaire HAL, est destinée au dépôt et à la diffusion de documents scientifiques de niveau recherche, publiés ou non, émanant des établissements d'enseignement et de recherche français ou étrangers, des laboratoires publics ou privés. 


\title{
A LINEARITY PRESERVING CELL-CENTERED SCHEME FOR THE HETEROGENEOUS AND ANISOTROPIC DIFFUSION EQUATIONS ON GENERAL MESHES*
}

\author{
ZHIMING $\mathrm{GAO}^{\dagger}$ AND JIMING $\mathrm{WU}^{\dagger}$
}

\begin{abstract}
In this paper a finite volume scheme for the heterogeneous and anisotropic diffusion equations is proposed on general, possibly nonconforming meshes. This scheme has both cell-centered unknowns and vertex unknowns. The vertex unknowns are treated as intermediate ones and are expressed as a linear weighted combination of the surrounding cell-centered unknowns, which reduces the scheme to a completely cell-centered one. We propose two types of new explicit weights which allow arbitrary diffusion tensors, and are neither discontinuity dependent nor mesh topology dependent. Both the derivation of the scheme and that of new weights satisfy the linearity preserving criterion which requires that a discretization scheme should be exact on linear solutions. The resulting new scheme is called as the linearity preserving cell-centered scheme and the numerical results show that it maintain optimal convergence rates for the solution and flux on general polygonal distorted meshes in case that the diffusion tensor is taken to be anisotropic, at times heterogeneous, and/or discontinuous.
\end{abstract}

Key words. diffusion equation, anisotropic diffusion tensor, cell-centered scheme, linearity preserving criterion, nonconforming mesh

AMS subject classifications. 65N08, 65N12

1. Introduction. This paper will focus on the investigation of new discretization scheme for solving the anisotropic steady-state diffusion equation

$$
\begin{gathered}
-\operatorname{div}(\boldsymbol{K} \nabla u)=f \text { in } \Omega, \\
u=g_{D} \text { on } \Gamma_{D}, \\
-\boldsymbol{K} \nabla u \cdot \mathbf{n}=g_{N} \text { on } \Gamma_{N},
\end{gathered}
$$

where $\boldsymbol{K}(x, y)$ is a $2 \times 2$ diffusion tensor, $f$ is the source term, $\Omega$ is a bounded polygonal domain in $R^{2}$ with its boundary $\partial \Omega=\bar{\Gamma}_{D} \cup \bar{\Gamma}_{N}$ where Dirichlet and Neumann boundary conditions are imposed on $\Gamma_{D}$ and $\Gamma_{N}$ respectively. $\mathbf{n}$ denotes the outward unit vector normal to the boundary $\partial \Omega$ and $g_{D}, g_{N}$ are given scalar functions which are almost everywhere defined on $\Gamma_{D}, \Gamma_{N}$ respectively.

Equations of this kind arise in a wide range of scientific fields such as hydrogeology, oil reservoir simulations, plasma physics, semiconductor modelling, Navier-Stokes equations, biology, and so on. For example, in porous media $u$ is called pressure, the diffusion tensor $\boldsymbol{K}$ may be highly anisotropic, heterogeneous and/or discontinuous, which imposes a great challenge for discretization schemes. In addition, another difficulty is that the computational mesh is usually nonconforming, highly distorted and highly skewed in some applications such as laser driven inertial confinement fusion.

The development of a discretization scheme should be based on the requirements motivated by both practical implementation and physical background, the issues about which range from the classical stability and accuracy to some other desirable numerical properties, local stencil, local conservation, monotonicity, allowing arbitrary diffusion tensors, simplicity, robustness, cost-efficiency, and so on. To our knowledge,

${ }^{*}$ This work is supported by the National Natural Science Foundation of China (No.10871030) and a grant from the Laboratory of Computational Physics.

${ }^{\dagger}$ Laboratory of Computational Physics, Institute of Applied Physics and Computational Mathematics, P. O. Box 8009-26, Beijing 100088, P. R. China (gao@iapcm.ac.cn wu_jiming@iapcm.ac.cn). 
there exists no scheme satisfying all the above properties. Among the aforementioned desirable properties, from our point of view, accuracy, cost-efficiency and allowing arbitrary diffusion tensors are among the fundamental ones.

We are interested in the discretization scheme which satisfies the so-called linearity preserving criterion, which requires that each step of the derivation of a discretization scheme for diffusion equation is exact or linearly exact, i.e., exact in the sense whenever the solution is a linear function and the diffusion coefficient is a constant tensor. We observe that some authors mentioned linearity preserving criterion in their works $[19,21,20,5,6]$. To our knowledge, the authors usually claimed that certain scheme satisfies or does not satisfy the linearity preserving criterion without intensive investigation. Although the linearity preserving criterion, at the present, has not been theoretically proved to be a sufficient or necessary condition for certain good numerical properties mentioned above, we observe from our numerical practice and other peoples' works that a discretization scheme under linearity preserving criterion usually maintains good accuracy on highly distorted meshes. Our point of view is confirmed once again by a recent work[5]. This scheme keeps all good numerical properties that a desirable mimetic finite difference (MFD) scheme can have, except the linearity preserving property. Numerical experiments done by the authors show that this scheme performs poorly on many distorted quadrilateral meshes. Motivated by the above observation, we will suggest a new discretization scheme under linearity preserving criterion in this paper.

In this paper, under the linearity preserving criterion, we propose a new finite volume scheme for the anisotropic diffusion equations on general meshes. In this scheme, the flux on each cell edge is explicitly expressed by two cell-centered unknowns defined on the cells sharing that edge, and two vertex unknowns at the two endpoints. A certain treatment for the vertex unknowns must be taken into consideration and the simplest and common way is to treat them as intermediate ones, more specifically, express them as certain linear combinations of the neighboring cell-centered unknowns. The coefficients in the linear combinations are known as the weights. To our knowledge, there exists in the literature some ways to find the weights for the vertex unknowns, such as by Taylor expansion [13, 24], by a straightforward bilinear interpolation [25] or its modification[4], by finite point method [17] or by some others[28]. Most of these methods are obtained in the isotropic case, and all these methods are either discontinuity dependent or mesh topology dependent. That a method is discontinuity dependent means that the derivation and display of the weights must be done under the condition that the location of the discontinuity is known beforehand. The implementation of a discontinuity dependent method will encounter difficulties and troubles when some moving discontinuity, such as shock wave, is presented. If a method for derivation of the weights is done under the condition that an interior vertex should meet four cell edges and its extension to the boundary vertex is not straightforward, it is called here mesh topology dependent. Obviously, it is difficult or complicated to extend a topology dependent method to the unstructured or nonconforming meshes. A linear least square approximation to calculate the weights are proposed in [8] and a generalization of this strategy for a more accurate reconstruction of the Neumann boundary vertex unknowns is developed in [18]. The weights in [18] is neither discontinuity dependent nor mesh topology dependent, but is not physically reasonable since the diffusion tensor is not taken into account.

Recently, a new approach in [22] has a desirable accuracy on highly distorted quadrilateral meshes, but it is a little complicate and involves a large amount of extra 
computational cost. A further improvement, with much reduced extra computational cost, was proposed in [26] by employing a multi-point flux approximation (MPFA) technique. These two types of weights are not discontinuity dependent and the later is even not mesh topology dependent, however, they are not given explicitly, instead, they are evaluated by solving certain local linear systems whose solvability itself is often proved to be a difficult problem. In addition, in the construction of other discretization schemes such as the local support operator scheme (LSOM)[20], the local flux mimetic finite difference scheme (LFMFD)[15], the physical space derived MPFA[1, 14] together with its variations[7], and the reference space derived MPFA[23, $2]$, the solution of certain local linear system is also required.

The above observations have motivated us to propose two types of new weights for the proposed scheme, and the solution of certain local linear systems is avoided. Our new scheme has the following characteristics:

(i) it has only cell-centered unknowns and a local stencil;

(ii) it satisfies the linearity preserving criterion;

(iii) it allows arbitrary diffusion tensors;

(iv) it leads to a nine-point scheme in the case of quadrilateral meshes;

(v) it is neither discontinuity dependent nor mesh topology dependent;

(vi) it has second order convergence rate for the approximate solution and higher than first order accuracy for the flux on general meshes;

(vii) the explicit weights in this scheme are easy to code and have low computational costs.

The rest of this paper is organized as follows. In Section 2, we derive the linearity preserving cell-centered scheme. The numerical results are presented in Section 3 to show the accuracy and efficiency of the linearity preserving cell-centered scheme and some conclusions are given in the last section.

2. Construction of the scheme. This section is devoted to the derivation of the new scheme under the linearity preserving criterion. Let $\boldsymbol{F}=-\boldsymbol{K} \nabla u$ denote the flux which satisfies the mass balance equation

$$
\operatorname{div} \boldsymbol{F}=f \text { in } \Omega .
$$

Integrating it over a polygon $e$ and using Gauss divergence theorem, we get

$$
\int_{\partial e} \boldsymbol{F} \cdot \mathbf{n}_{\mathbf{e}} \mathrm{d} s=\iint_{e} f(x, y) \mathrm{d} x \mathrm{~d} y
$$

where $\mathbf{n}_{e}$ denotes the outward unit vector normal to the cell boundary $\partial e$.

In this paper, we shall derive a discrete expression for the integration of $\boldsymbol{F} \cdot \mathbf{n}_{\mathbf{e}}$ on each cell edge. Throughout this section we assume that

(i) = is used if the derivation is exact;

(ii) $\simeq$ is used if the approximation satisfies the linearity preserving criterion;

(iii) $\approx$ is used when an approximation is not subjected to the linearity preserving criterion.

2.1. Discretization of the diffusion flux. This subsection is devoted to derive the expression for the flux on each cell edge under the linearity preserving criterion. We begin the derivation by introducing the following straightforward result.

LEMMA 2.1. Let $\triangle O P Q$ be a triangle with vertices $O, P, Q$ ordered counterclockwise. For a function u defined on $\triangle O P Q$, we have

$$
\nabla u \simeq \frac{u_{Q}-u_{P}}{|P Q|^{2}} \overrightarrow{P Q}+\frac{\mathcal{R} \overrightarrow{P Q}}{|P Q|^{2}}\left[\left(u_{P}-u_{O}\right) \cot \angle P Q O+\left(u_{Q}-u_{O}\right) \cot \angle O P Q\right],
$$


where $S_{\triangle O P Q}$ is the area of $\triangle O P Q, u_{O}, u_{P}, u_{Q}$ are the values of $u$ at $O, P, Q$ respectively, and $\mathcal{R}$ denotes a vector operator which rotates a vector clockwise to its normal direction.

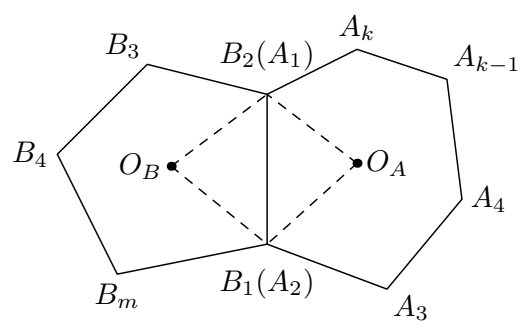

Fig. 2.1. A local diagram of the polygonal mesh.

Now, we suppose that the computational domain $\Omega$ is partitioned into a number of non-overlapping regular polygonal cells. As shown in Fig. 2.1, we shall use the following notations to derive our scheme:

(i) $O_{A}$ is the cell center of an arbitrary cell $A_{1} A_{2} \cdots A_{k}, \boldsymbol{n}_{A}$ is the outward unit vector normal to the cell boundary $\partial A_{1} A_{2} \cdots A_{k}, h_{A}^{(i)}$ is the distance from $O_{A}$ to $A_{i} A_{i+1}$ with $A_{k+1}=A_{1}$, and the notations $O_{B}, \mathbf{n}_{B}, h_{B}^{i}$, and $B_{m+1}$ for the neighboring cell $B_{1} B_{2} \cdots B_{m}$ can be analogously introduced.

(ii) $\boldsymbol{K}_{\alpha}=\boldsymbol{K}\left(x_{O_{\alpha}}, y_{O_{\alpha}}\right), \quad \alpha=A, B$.

(iii) $u_{A}, u_{B}$ are the approximations of $u$ on two cells $A_{1} A_{2} \cdots A_{k}$ and $B_{1} B_{2} \cdots B_{m}$ respectively, and $u_{A_{i}}, u_{B_{i}}$ denote the approximations of $u$ at the vertices $A_{i}, B_{i}$.

To begin with, we derive the discrete flux across an internal edge. Using Lemma 2.1 on the triangle $O_{B} B_{1} B_{2}$, we obtain

$$
\begin{aligned}
\nabla u \simeq \frac{\mathcal{R} \overrightarrow{B_{1} B_{2}}}{\left|B_{1} B_{2}\right|^{2}}\left[\left(u_{B_{1}}-u_{B}\right)\right. & \cot \angle B_{1} B_{2} O_{B} \\
& \left.\quad+\left(u_{B_{2}}-u_{B}\right) \cot \angle O_{B} B_{1} B_{2}\right]+\left(u_{B_{2}}-u_{B_{1}}\right) \frac{\overrightarrow{B_{1} B_{2}}}{\left|B_{1} B_{2}\right|^{2}} .
\end{aligned}
$$

Then

$$
\begin{aligned}
\boldsymbol{F} \cdot\left(\mathcal{R} \overrightarrow{B_{1} B_{2}}\right) \simeq-K_{B}^{(n)}\left[\left(u_{B_{1}}-u_{B}\right) \cot \angle B_{1} B_{2} O_{B}\right. \\
\\
\left.\quad+\left(u_{B_{2}}-u_{B}\right) \cot \angle O_{B} B_{1} B_{2}\right]-\left(u_{B_{2}}-u_{B_{1}}\right) K_{B}^{(t)},
\end{aligned}
$$

where

$$
K_{\alpha}^{(n)}=\frac{\left(\mathcal{R} \overrightarrow{B_{1} B_{2}}\right)^{T} \boldsymbol{K}_{\alpha}\left(\mathcal{R} \overrightarrow{B_{1} B_{2}}\right)}{\left|B_{1} B_{2}\right|^{2}}, \quad K_{\alpha}^{(t)}=\frac{\left(\mathcal{R} \overrightarrow{B_{1} B_{2}}\right)^{T} \boldsymbol{K}_{\alpha} \overrightarrow{B_{1} B_{2}}}{\left|B_{1} B_{2}\right|^{2}}, \alpha=A, B .
$$

It follows that

$$
\begin{aligned}
& \frac{h_{B}^{(1)}}{K_{B}^{(n)}} \int_{B_{1} B_{2}} \boldsymbol{F} \cdot \boldsymbol{n}_{B} \mathrm{~d} s \simeq-\frac{1}{\left|B_{1} B_{2}\right|}\left[\left(\overrightarrow{B_{2} O_{B}} \cdot \overrightarrow{B_{2} B_{1}}\right)\left(u_{B_{1}}-u_{B}\right)\right. \\
&\left.+\left(\overrightarrow{B_{1} O_{B}} \cdot \overrightarrow{B_{1} B_{2}}\right)\left(u_{B_{2}}-u_{B}\right)\right]-\left(u_{B_{2}}-u_{B_{1}}\right) \frac{h_{B}^{(1)} K_{B}^{(t)}}{K_{B}^{(n)}}
\end{aligned}
$$


Analogously on the triangular domain $O_{A} A_{1} A_{2}$,

$$
\begin{aligned}
& \frac{h_{A}^{(1)}}{K_{A}^{(n)}} \int_{B_{1} B_{2}} \boldsymbol{F} \cdot \boldsymbol{n}_{A} \mathrm{~d} s \simeq-\frac{1}{\left|B_{1} B_{2}\right|}\left[\left(\overrightarrow{B_{1} O_{A}} \cdot \overrightarrow{B_{1} B_{2}}\right)\left(u_{B_{2}}-u_{A}\right)\right. \\
&\left.+\left(\overrightarrow{B_{2} O_{A}} \cdot \overrightarrow{B_{2} B_{1}}\right)\left(u_{B_{1}}-u_{A}\right)\right]-\left(u_{B_{1}}-u_{B_{2}}\right) \frac{h_{A}^{(1)} K_{A}^{(t)}}{K_{A}^{(n)}}
\end{aligned}
$$

Since the flux is continuous across the cell edge $B_{1} B_{2}$,

$$
\int_{B_{1} B_{2}} \boldsymbol{F} \cdot \boldsymbol{n}_{A} \mathrm{~d} s+\int_{B_{1} B_{2}} \boldsymbol{F} \cdot \boldsymbol{n}_{B} \mathrm{~d} s=0
$$

from (2.5), we obtain

$$
\begin{aligned}
& \frac{h_{A}^{(1)}}{K_{A}^{(n)}} \int_{B_{1} B_{2}} \boldsymbol{F} \cdot \boldsymbol{n}_{B} \mathrm{~d} s \simeq \frac{1}{\left|B_{1} B_{2}\right|}\left[\left(\overrightarrow{B_{1} O_{A}} \cdot \overrightarrow{B_{1} B_{2}}\right)\left(u_{B_{2}}-u_{A}\right)\right. \\
&\left.+\left(\overrightarrow{B_{2} O_{A}} \cdot \overrightarrow{B_{2} B_{1}}\right)\left(u_{B_{1}}-u_{A}\right)\right]+\left(u_{B_{1}}-u_{B_{2}}\right) \frac{h_{A}^{(1)} K_{A}^{(t)}}{K_{A}^{(n)}}
\end{aligned}
$$

Finally, by taking an average from (2.4) and (2.6), we have the following discretization of the flux on the interior edge $B_{1} B_{2}$ :

$$
\int_{B_{1} B_{2}} \boldsymbol{F} \cdot \boldsymbol{n}_{B} \mathrm{~d} s \simeq-\mathcal{K}_{A B}^{(1)}\left|B_{1} B_{2}\right|\left[u_{A}-u_{B}-\mathcal{D}_{A B}^{(1)}\left(u_{B_{2}}-u_{B_{1}}\right)\right]
$$

where

$\mathcal{K}_{A B}^{(1)}=\frac{K_{A}^{(n)} K_{B}^{(n)}}{K_{A}^{(n)} h_{B}^{(1)}+K_{B}^{(n)} h_{A}^{(1)}}, \mathcal{D}_{A B}^{(1)}=\frac{\overrightarrow{B_{1} B_{2}} \cdot \overrightarrow{O_{B} O_{A}}}{\left|B_{1} B_{2}\right|^{2}}-\frac{1}{\left|B_{1} B_{2}\right|}\left(\frac{K_{B}^{(t)}}{K_{B}^{(n)}} h_{B}^{(1)}+\frac{K_{A}^{(t)}}{K_{A}^{(n)}} h_{A}^{(1)}\right)$.

The expressions of the flux on other edges in the domain can be derived similarly. If $B_{1} B_{2}$ is located on the Dirichlet boundary, we obtain directly from (2.4)

$$
\begin{aligned}
\int_{B_{1} B_{2}} \boldsymbol{F} & \cdot \boldsymbol{n}_{B} \mathrm{~d} s \simeq-\frac{K_{B}^{(n)}}{h_{B}^{(1)}\left|B_{1} B_{2}\right|}\left[\left(\overrightarrow{B_{2} O_{B}} \cdot \overrightarrow{B_{2} B_{1}}\right) g_{D}\left(B_{1}\right)\right. \\
& \left.+\left(\overrightarrow{B_{1} O_{B}} \cdot \overrightarrow{B_{1} B_{2}}\right) g_{D}\left(B_{2}\right)-\left|B_{1} B_{2}\right|^{2} u_{B}\right]-\left[g_{D}\left(B_{2}\right)-g_{D}\left(B_{1}\right)\right] K_{B}^{(t)}
\end{aligned}
$$

where $g_{D}\left(B_{1}\right)$ and $g_{D}\left(B_{2}\right)$ are the values of Dirichlet boundary function $g_{D}$ at the vertices $B_{1}$ and $B_{2}$ respectively.

Finally we conclude that the discrete flux across an edge $B_{1} B_{2}$ is

$$
\int_{B_{1} B_{2}} \boldsymbol{F} \cdot \boldsymbol{n}_{B} \mathrm{~d} s \simeq \begin{cases}\text { formula }(2.7), & \text { if } B_{1} B_{2} \text { is an interior edge, } \\ \text { formula }(2.8), & \text { if } B_{1} B_{2} \text { is located on } \Gamma_{D} \\ \int_{B_{1} B_{2}} g_{N} \mathrm{~d} s, & \text { if } B_{1} B_{2} \text { is located on } \Gamma_{N} .\end{cases}
$$

REMARK 2.1. In a structured quadrilateral mesh, the discrete flux (2.7) will result in a nine-point scheme which has certain relationship with some existing schemes: 
(i) The tangential term in $\mathcal{D}_{A B}^{(1)}$ disappears if $\boldsymbol{K}$ is isotropic. In this case, the expression for the discrete flux in (2.7) reduces to that in [16, 26, 28];

(ii) If $\boldsymbol{K}_{A}=\boldsymbol{K}_{B}=\boldsymbol{K}_{B_{1} B_{2}}$ with $\boldsymbol{K}_{B_{1} B_{2}}$ being defined as certain mean diffusion tensor on $B_{1} B_{2}$, (2.7) leads to that in [18].

The vertex unknown in the discrete flux (2.9) will be eliminated by expressing it as a linear combination of the neighboring cell-centered unknowns, which will be discussed in the subsequent subsections. The treatment for the vertex unknown is very important since it has a great influence on the accuracy of our scheme.

2.2. The treatments for the interior vertex unknowns. This section is devoted to the study of the weights for the interior vertex unknowns, whereas the treatments for boundary vertex unknowns will be given in subsection 2.3.

As shown in Fig. 2.2(a), a vertex unknown can be expressed as

$$
u_{0}=\sum_{k=1}^{\mathcal{N}\left(Q_{0}\right)} \omega_{k} u_{k},
$$

where $u_{0}$ is the vertex unknown at the vertex $Q_{0}$ which is surrounded by cells $\Omega_{k}$, $\mathcal{N}\left(Q_{0}\right)$ is the number of cells sharing $Q_{0}, u_{k}$ is the value of $u$ at the center $O_{k}$ of $\Omega_{k}$ which is enclosed by cell edges $Q_{0} P_{k}$ and $Q_{0} P_{k+1}$, and $\omega_{k}$ is known as the weight.

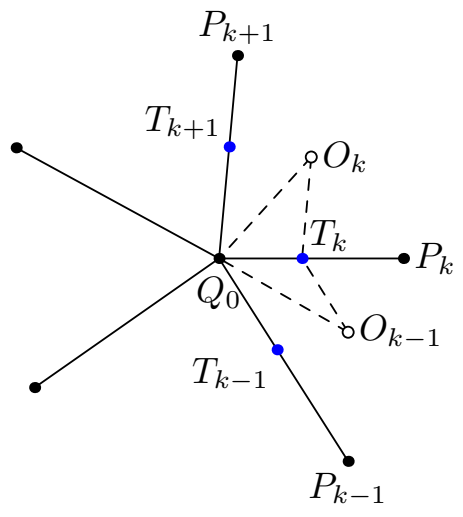

(a)

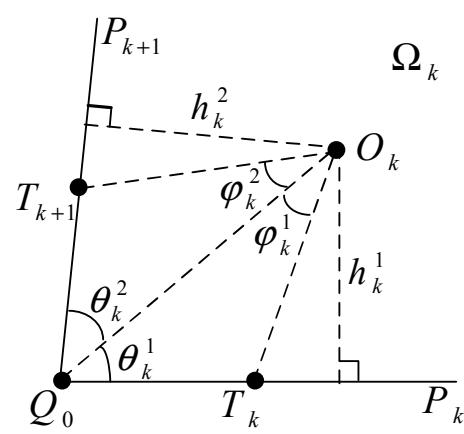

(b)

Fig. 2.2. Notations around an interior vertex $Q_{0}$.

2.2.1. The first type of new weights with explicit expressions. As depicted in Fig. 2.2, $T_{k}$ is a dynamic point on the edge $Q_{0} P_{k}$, defined by

$$
T_{k}=\tau\left(Q_{0}, k\right) Q_{0}+\left[1-\tau\left(Q_{0}, k\right)\right] P_{k}, \quad k=1, \cdots, \mathcal{N}\left(Q_{0}\right), 0<\tau\left(Q_{0}, k\right)<1,
$$

where $\tau\left(Q_{0}, k\right)$ is a dynamic parameter, dependent of $Q_{0}$ and the edges sharing $Q_{0} \cdot \bar{u}_{k}$ is the unknowns defined at $T_{k}$, Let $h_{k}^{1}, h_{k}^{2}$ be the distances from $O_{k}$ to $Q_{0} P_{k}, Q_{0} P_{k+1}$ respectively. The rest notations $\theta_{k}^{1}, \theta_{k}^{2}, \varphi_{k}^{1}, \varphi_{k}^{2}$ are depicted in Fig. 2.2(b).

For simplicity, we define $\boldsymbol{K}_{k}=\boldsymbol{K}\left(x_{O_{k}}, y_{O_{k}}\right)$ and $\eta_{k}^{i}=\left|Q_{0} T_{k+i-1}\right|^{2} / h_{k}^{i}, i=$ 1,2 . We shall also employ a periodic numbering for the cells and vertices such as $P_{\mathcal{N}\left(Q_{0}\right)+1}=P_{1}, P_{0}=P_{\mathcal{N}\left(Q_{0}\right)}, T_{0}=T_{\mathcal{N}\left(Q_{0}\right)}$, etc. Moreover, in the following discussion, we shall only consider the case where $\tau\left(Q_{0}, k\right)=\tau\left(Q_{0}\right)$, i.e., the dynamic 
parameter depends only on $Q_{0}$ and assumes the same value on the edges sharing $Q_{0}$. Besides, we shall drop $\left(Q_{0}\right)$ and simply use $\tau$ whenever there is no ambiguity.

Now, using Lemma 2.1 on triangle domain $\triangle O_{k} Q_{0} T_{k}$, we have

$$
\nabla u \simeq \frac{\bar{u}_{k}-u_{0}}{\left|Q_{0} T_{k}\right|^{2}} \overrightarrow{Q_{0} T_{k}}+\frac{\mathcal{R} \overrightarrow{Q_{0} T_{k}}}{\left|Q_{0} T_{k}\right|^{2}}\left[\left(u_{0}-u_{k}\right) \cot \angle Q_{0} T_{k} O_{k}+\left(\bar{u}_{k}-u_{k}\right) \cot \theta_{k}^{1}\right],
$$

which leads to

$$
\boldsymbol{F} \cdot\left(\mathcal{R} \overrightarrow{Q_{0} T_{k}}\right) \simeq K_{k}^{(n), 1} \eta_{k}^{1}\left(u_{k}-u_{0}\right)-\left(K_{k}^{(t), 1}+\cot \theta_{k}^{1} K_{k}^{(n), 1}\right)\left(\bar{u}_{k}-u_{0}\right),
$$

where for $\alpha=1,2, \cdots, \mathcal{N}\left(Q_{0}\right)$ and $i=1,2$,

$$
K_{\alpha}^{(n), i}=\frac{\left(\mathcal{R} \overrightarrow{Q_{0} T_{\alpha+i-1}}\right)^{T} \boldsymbol{K}_{\alpha}\left(\mathcal{R} \overrightarrow{Q_{0} T_{\alpha+i-1}}\right)}{\left|Q_{0} T_{\alpha+i-1}\right|^{2}}, K_{\alpha}^{(t), i}=\frac{\left(\mathcal{R} \overrightarrow{Q_{0} T_{\alpha+i-1}}\right)^{T} \boldsymbol{K}_{\alpha} \overrightarrow{Q_{0} T_{\alpha+i-1}}}{\left|Q_{0} T_{\alpha+i-1}\right|^{2}} .
$$

Similarly, on the triangle $\triangle O_{k-1} T_{k} Q_{0}$,

$$
\boldsymbol{F} \cdot\left(\mathcal{R} \overrightarrow{T_{k} Q_{0}}\right) \simeq K_{k-1}^{(n), 2} \eta_{k-1}^{2}\left(u_{k-1}-u_{0}\right)+\left(K_{k-1}^{(t), 2}-\cot \theta_{k-1}^{2} K_{k-1}^{(n), 2}\right)\left(\bar{u}_{k}-u_{0}\right) .
$$

By noting

$$
\boldsymbol{F} \cdot\left(\mathcal{R} \overrightarrow{Q_{0} T_{k}}\right)+\boldsymbol{F} \cdot\left(\mathcal{R} \overrightarrow{T_{k} Q_{0}}\right)=0
$$

and through some algebra, we obtain

$$
\bar{u}_{k}-u_{0}=\frac{K_{k}^{(n), 1} \eta_{k}^{1}\left(u_{k}-u_{0}\right)+K_{k-1}^{(n), 2} \eta_{k-1}^{2}\left(u_{k-1}-u_{0}\right)}{K_{k-1}^{(n), 2} \cot \theta_{k-1}^{2}+K_{k}^{(n), 1} \cot \theta_{k}^{1}-K_{k-1}^{(t), 2}+K_{k}^{(t), 1}} .
$$

Now we have obtained $\mathcal{N}\left(Q_{0}\right)$ equations for $\bar{u}_{k}\left(k=1, \cdots, \mathcal{N}\left(Q_{0}\right)\right)$ and $u_{0}$. In order to eliminate all the intermediate unknowns, we should derive one more equation by the linearity preserving criterion. When the solution is a piecewise linear function and the diffusion tensor is constant, the contour integration of normal flux along the boundaries of quadrilateral subcells $Q_{0} T_{k} O_{k} T_{k+1}\left(k=1, \cdots, \mathcal{N}\left(Q_{0}\right)\right)$ are all zero. Hence, by using the flux continuity condition (2.12), we obtain

$$
\sum_{k=1}^{\mathcal{N}\left(Q_{0}\right)}\left[\boldsymbol{F} \cdot\left(\mathcal{R} \overrightarrow{T_{k} O_{k}}\right)+\boldsymbol{F} \cdot\left(\mathcal{R} \overrightarrow{O_{k-1} T_{k}}\right)\right] \simeq 0
$$

Similar to the derivation of (2.11), we have

$$
\begin{aligned}
\boldsymbol{F} \cdot\left(\mathcal{R} \overrightarrow{T_{k} O_{k}}\right) \simeq\left(\bar{u}_{k}-u_{0}\right)\left[\tilde{K}_{k}^{(t), 1}-\tilde{K}_{k}^{(n), 1} \cot \varphi_{k}^{1}\right] & \\
& +\left(u_{k}-u_{0}\right)\left[\tilde{K}_{k}^{(n), 1} \cot \left(\theta_{k}^{1}+\varphi_{k}^{1}\right)-\tilde{K}_{k}^{(t), 1}\right]
\end{aligned}
$$

and

$$
\begin{aligned}
\boldsymbol{F} \cdot\left(\mathcal{R} \overrightarrow{O_{k-1} T_{k}}\right) \simeq\left(u_{k-1}-u_{0}\right)\left[\tilde{K}_{k-1}^{(t), 2}+\tilde{K}_{k-1}^{(n), 2} \cot \left(\theta_{k-1}^{2}+\varphi_{k-1}^{2}\right)\right] \\
\\
-\left(\bar{u}_{k}-u_{0}\right)\left[\tilde{K}_{k-1}^{(t), 2}+\tilde{K}_{k-1}^{(n), 2} \cot \varphi_{k-1}^{2}\right],
\end{aligned}
$$


where in the cell $\Omega_{\alpha}, \alpha=1,2, \cdots, \mathcal{N}\left(Q_{0}\right)$, for $i=0,1$ we define

$$
\tilde{K}_{\alpha}^{(n), i+1}=\frac{\left(\mathcal{R} \overrightarrow{T_{\alpha+i} O_{\alpha}}\right)^{T} \boldsymbol{K}_{\alpha}\left(\mathcal{R} \overrightarrow{T_{\alpha+i} O_{\alpha}}\right)}{\left|T_{\alpha+i} O_{\alpha}\right|^{2}}, \tilde{K}_{\alpha}^{(t), i+1}=\frac{\left(\mathcal{R} \overrightarrow{T_{\alpha+i} O_{\alpha}}\right)^{T} \boldsymbol{K}_{\alpha} \overrightarrow{T_{\alpha+i} O_{\alpha}}}{\left|T_{\alpha+i} O_{\alpha}\right|^{2}} .
$$

From (2.14), the $\left(\mathcal{N}\left(Q_{0}\right)+1\right)$-th equation follows,

$$
\begin{aligned}
\sum_{k=1}^{\mathcal{N}\left(Q_{0}\right)}\left[\tilde{K}_{k-1}^{(n), 2}\right. & \left.\cot \varphi_{k-1}^{2}+\tilde{K}_{k}^{(n), 1} \cot \varphi_{k}^{1}+\tilde{K}_{k-1}^{(t), 2}-\tilde{K}_{k}^{(t), 1}\right]\left(\bar{u}_{k}-u_{0}\right) \\
- & {\left[\sum_{i=1}^{2} \tilde{K}_{k}^{(n), i} \cot \left(\theta_{k}^{i}+\varphi_{k}^{i}\right)-\tilde{K}_{k}^{(t), 1}+\tilde{K}_{k}^{(t), 2}\right]\left(u_{k}-u_{0}\right) \simeq 0 . }
\end{aligned}
$$

Substituting (2.13) into the above equation and letting

$$
\lambda_{k}=K_{k}^{(n), 1} \eta_{k}^{1} \phi(k)+K_{k}^{(n), 2} \eta_{k}^{2} \phi(k+1)-\sum_{i=1}^{2} \tilde{K}_{k}^{(n), i} \cot \left(\theta_{k}^{i}+\varphi_{k}^{i}\right)+\tilde{K}_{k}^{(t), 1}-\tilde{K}_{k}^{(t), 2}
$$

with

$$
\phi(k)=\frac{\tilde{K}_{k-1}^{(n), 2} \cot \varphi_{k-1}^{2}+\tilde{K}_{k}^{(n), 1} \cot \varphi_{k}^{1}+\tilde{K}_{k-1}^{(t), 2}-\tilde{K}_{k}^{(t), 1}}{K_{k-1}^{(n), 2} \cot \theta_{k-1}^{2}+K_{k}^{(n), 1} \cot \theta_{k}^{1}-K_{k-1}^{(t), 2}+K_{k}^{(t), 1}},
$$

we obtain the new weights as follows

$$
\omega_{k}=\frac{\lambda_{k}}{\sum_{k=1}^{\mathcal{N}\left(Q_{0}\right)} \lambda_{k}}
$$

2.2.2. The second type of new weights with explicit expressions. In the above derivation, we have seen that the $\mathcal{N}\left(Q_{0}\right)+1$-th equation (2.15), which plays an important role in determining the new weights. By choosing a different contour, we can obtain another type of new weights. In fact, when the solution is a piecewise linear function and the diffusion coefficient is constant, the contour integration of the normal flux along the boundaries of triangles $Q_{0} T_{k} T_{k+1}\left(k=1,2, \cdots, \mathcal{N}\left(Q_{0}\right)\right)$ vanishes. Hence by using the flux continuity condition (2.12), we have

$$
\sum_{k=1}^{\mathcal{N}\left(Q_{0}\right)} \boldsymbol{F} \cdot\left(\mathcal{R} \overline{T_{k} T_{k+1}}\right) \simeq 0
$$

Applying Lemma 2.1 on triangle $Q_{0} T_{k} T_{k+1}$ yields

$\boldsymbol{F} \cdot \mathcal{R}\left({\overrightarrow{T_{k} T}}_{k+1}\right) \simeq\left(\bar{K}_{k}^{(t)}-\bar{K}_{k}^{(n)} \cot \vartheta_{k}^{2}\right)\left(\bar{u}_{k}-u_{0}\right)-\left(\bar{K}_{k}^{(t)}+\bar{K}_{k}^{(n)} \cot \vartheta_{k}^{1}\right)\left(\bar{u}_{k+1}-u_{0}\right)$,

where $\vartheta_{k}^{1}=\angle Q_{0} T_{k} T_{k+1}, \vartheta_{k}^{2}=\angle Q_{0} T_{k+1} T_{k}$ and at the cell $\Omega_{\alpha}, \alpha=1, \cdots, \mathcal{N}\left(Q_{0}\right)$,

$$
\bar{K}_{\alpha}^{(n)}=\frac{\left(\mathcal{R}{\overrightarrow{T_{\alpha} T_{\alpha+1}}}^{T} \boldsymbol{K}_{\alpha}\left(\mathcal{R} \overrightarrow{T_{\alpha} T_{\alpha+1}}\right)\right.}{\left|T_{\alpha} T_{\alpha+1}\right|^{2}}, \quad \bar{K}_{\alpha}^{(t)}=\frac{\left(\mathcal{R} \overrightarrow{T_{\alpha} T_{\alpha+1}}\right)^{T} \boldsymbol{K}_{\alpha} \overrightarrow{T_{\alpha} T_{\alpha+1}}}{\left|T_{\alpha} T_{\alpha+1}\right|^{2}} .
$$

It follows from (2.19) that

$$
\sum_{k=1}^{\mathcal{N}\left(Q_{0}\right)}\left[\bar{K}_{k-1}^{(t)}-\bar{K}_{k}^{(t)}+\bar{K}_{k-1}^{(n)} \cot \vartheta_{k-1}^{1}+\bar{K}_{k}^{(n)} \cot \vartheta_{k}^{2}\right]\left(\bar{u}_{k}-u_{0}\right) \simeq 0 .
$$


By substituting (2.13) into (2.20), we obtain another type of new weights

$$
w_{k}=\frac{\bar{\lambda}_{k}}{\sum_{k=1}^{\mathcal{N}\left(Q_{0}\right)} \bar{\lambda}_{k}}
$$

where

$$
\bar{\lambda}_{k}=K_{k}^{(n), 1} \eta_{k}^{1} \zeta(k)+K_{k}^{(n), 2} \eta_{k}^{2} \zeta(k+1)
$$

and

$$
\zeta(k)=\frac{\bar{K}_{k-1}^{(n)} \cot \vartheta_{k-1}^{1}+\bar{K}_{k}^{(n)} \cot \vartheta_{k}^{2}+\bar{K}_{k-1}^{(t)}-\bar{K}_{k}^{(t)}}{K_{k-1}^{(n), 2} \cot \theta_{k-1}^{2}+K_{k}^{(n), 1} \cot \theta_{k}^{1}-K_{k-1}^{(t), 2}+K_{k}^{(t), 1}} .
$$

Finally, this subsection is concluded by the following result and the proof is straightforward.

THEOREM 2.2. The new weights defined by (2.18) and (2.16) are dependent of the parameter $\tau$ in (2.10) whereas the new weights defined by (2.21) and (2.22) are independent of $\tau$.

2.3. The treatments for the boundary vertex unknowns. In this subsection we will explain how the boundary vertex value depdends on the approximate cell-centered unknowns. If one vertex $Q_{0}$ is a Dirichlet boundary vertex, one can simply set $u_{0}=g_{D}\left(Q_{0}\right)$. In this section we will focus on the treatment for the Nuemann boundary conditions. It will be clear that the treatment for boundary vertex unknowns has no essential difference with that for interior vertex unknowns.

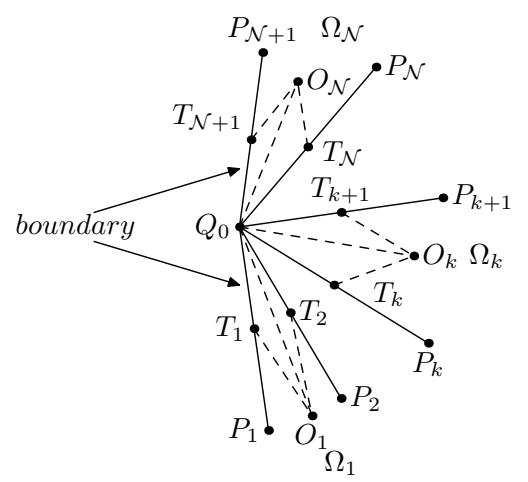

Fig. 2.3. Notations around a boundary vertex $Q_{0}$.

We assume that vertex $Q_{0}$ is located on a Nuemann boundary $\Gamma_{N}$ and define a local numbering for the cells and edges which are connected to $Q_{0}$ (see Fig. 2.3). $Q_{0}$ is surrounded by $\mathcal{N}$ cells, therefore there are $\mathcal{N}+1$ edges, the first and last edges are located on the boundary.

Firstly, by using the flux continuity across the cell edge $Q_{0} P_{k}$, we obtain (2.13) with $k=2, \cdots, \mathcal{N}$. Secondly, by applying Lemma 2.1 on triangle domain $\triangle O_{1} Q_{0} T_{1}$ and $\triangle O_{\mathcal{N}} T_{\mathcal{N}+1} Q_{0}$ respectively, we get

$$
\begin{aligned}
\bar{u}_{1}-u_{0} & \simeq \frac{K_{1}^{(n)} \eta_{1}^{1}\left(u_{1}-u_{0}\right)-\left|Q_{0} T_{1}\right| g_{N}\left(T_{1}\right)}{K_{1}^{(t)}+K_{1}^{(n)} \cot \theta_{1}^{1}} \\
\bar{u}_{\mathcal{N}+1}-u_{0} & \simeq \frac{K_{\mathcal{N}}^{(n)} \eta_{\mathcal{N}}^{2}\left(u_{\mathcal{N}}-u_{0}\right)-\left|Q_{0} T_{\mathcal{N}+1}\right| g_{N}\left(T_{\mathcal{N}+1}\right)}{K_{\mathcal{N}}^{(n)} \cot \theta_{\mathcal{N}}^{2}-K_{\mathcal{N}}^{(t)}}
\end{aligned}
$$


where $g_{N}\left(T_{1}\right)$ and $g_{N}\left(T_{\mathcal{N}+1}\right)$ denote the values of $g_{N}$ at $T_{1}$ and $T_{\mathcal{N}+1}$, respectively.

Finally, the last equation is obtained in a way which is similar to that of (2.15). By choosing the contour $Q_{0} \rightarrow T_{1} \rightarrow O_{1} \rightarrow \cdots \cdots \rightarrow T_{\mathcal{N}+1} \rightarrow Q_{0}$, we obtain

$$
\sum_{k=1}^{\mathcal{N}}\left[\boldsymbol{F} \cdot\left(\mathcal{R} \overrightarrow{T_{k} O_{k}}\right)+\boldsymbol{F} \cdot\left(\mathcal{R} \overrightarrow{O_{k} T_{k+1}}\right)\right]+\boldsymbol{F} \cdot\left(\mathcal{R} \overrightarrow{Q_{0} T_{1}}\right)+\boldsymbol{F} \cdot\left(\mathcal{R} \overrightarrow{T_{\mathcal{N}+1} Q_{0}}\right) \simeq 0
$$

By Lemma 2.1, we have

$$
\begin{array}{r}
\sum_{k=1}^{\mathcal{N}}\left\{\left(\bar{u}_{k}-u_{0}\right)\left[\tilde{K}_{k}^{(t), 1}-\tilde{K}_{k}^{(n), 1} \cot \varphi_{k}^{1}\right]-\left(\bar{u}_{k+1}-u_{0}\right)\left[\tilde{K}_{k}^{(n), 2} \cot \varphi_{k}^{2}+\tilde{K}_{k}^{(t), 2}\right]\right\} \\
+\sum_{k=1}^{\mathcal{N}}\left[\tilde{K}_{k}^{(n), 1} \cot \left(\theta_{k}^{1}+\varphi_{k}^{1}\right)+\tilde{K}_{k}^{(n), 2} \cot \left(\theta_{k}^{2}+\varphi_{k}^{2}\right)-\tilde{K}_{k}^{(t), 1}+\tilde{K}_{k}^{(t), 2}\right]\left(u_{k}-u_{0}\right) \\
+\left|Q_{0} T_{1}\right| g_{N}\left(T_{1}\right)+\left|Q_{0} T_{\mathcal{N}+1}\right| g_{N}\left(T_{\mathcal{N}+1}\right) \simeq 0 .
\end{array}
$$

Substituting (2.13), (2.24) and (2.25) into the above equation, we get the first type of explicit weights for the boundary vertex unknown $u_{0}$

$$
u_{0} \simeq\left(\sum_{k=1}^{\mathcal{N}} \lambda_{k}\right)^{-1}\left\{\sum_{k=1}^{\mathcal{N}} \lambda_{k} u_{k}-\sum_{j=1, \mathcal{N}+1}[1+\phi(j)]\left|Q_{0} T_{j}\right| g_{N}\left(T_{j}\right)\right\},
$$

where $\lambda_{k}$ is defined by (2.16) and

$$
\phi(k)= \begin{cases}\frac{\tilde{K}_{1}^{(n), 1} \cot \varphi_{1}^{1}-\tilde{K}_{1}^{(t), 1}}{K_{1}^{(n)} \cot \theta_{1}^{1}+K_{1}^{(t)}}, & k=1 ; \\ \text { formula }(2.17), & 2 \leq k \leq \mathcal{N} ; \\ \frac{\tilde{K}_{\mathcal{N}}^{(n), 2} \cot \varphi_{\mathcal{N}}^{2}+\tilde{K}_{\mathcal{N}}^{(t), 2}}{K_{\mathcal{N}}^{(n)} \cot \theta_{\mathcal{N}}^{2}-K_{\mathcal{N}}^{(t)}}, & k=\mathcal{N}+1 .\end{cases}
$$

We can also choose another contour as $Q_{0} \rightarrow T_{1} \rightarrow \cdots \rightarrow T_{\mathcal{N}+1} \rightarrow Q_{0}$ and get

$$
\begin{aligned}
\sum_{k=1}^{\mathcal{N}}\left[\left(\bar{K}_{k}^{(t)}-\bar{K}_{k}^{(n)} \cot \vartheta_{k}^{2}\right)\left(\bar{u}_{k}-u_{0}\right)\right. & \left.-\left(\bar{K}_{k}^{(t)}+\bar{K}_{k}^{(n)} \cot \vartheta_{k}^{1}\right)\left(\bar{u}_{k+1}-u_{0}\right)\right] \\
& +\left|Q_{0} T_{1}\right| g_{N}\left(T_{1}\right)+\left|Q_{0} T_{\mathcal{N}+1}\right| g_{N}\left(T_{\mathcal{N}+1}\right) \simeq 0 .
\end{aligned}
$$

Substituting (2.13), (2.24) and (2.25) into the above equation, we get another type of explicit weights for the boundary vertex $u_{0}$

$$
u_{0} \simeq\left(\sum_{k=1}^{\mathcal{N}} \bar{\lambda}_{k}\right)^{-1}\left[\sum_{k=1}^{\mathcal{N}} \bar{\lambda}_{k} u_{k}-\sum_{j=1, \mathcal{N}+1}[1+\zeta(j)]\left|Q_{0} T_{j}\right| g_{N}\left(T_{j}\right)\right],
$$

where $\bar{\lambda}_{k}$ is defined by (2.22) and

$$
\zeta(k)= \begin{cases}\frac{\bar{K}_{1}^{(n)} \cot \vartheta_{1}^{2}-\bar{K}_{1}^{(t)}}{K_{1}^{(n)} \cot \theta_{1}^{1}+K_{1}^{(t)}}, & k=1 ; \\ \text { formula }(2.23), & 2 \leq k \leq \mathcal{N} ; \\ \frac{\bar{K}_{\mathcal{N}}^{(n)} \cot \vartheta_{\mathcal{N}}^{1}+\bar{K}_{\mathcal{N}}^{(t)}}{K_{\mathcal{N}}^{(n)} \cot \theta_{\mathcal{N}}^{2}-K_{\mathcal{N}}^{(t)}}, & k=\mathcal{N}+1 .\end{cases}
$$


3. Numerical Examples. In this section, we present some numerical results to demonstrate the accuracy and the efficiency of the new scheme on various meshes. The notations of the algorithms used in this section are displayed in Table 3.1.

All tests presented in this section have analytical solutions, so the solution errors and edge normal flux errors are investigated in the discrete $L^{2}$ norms, which are defined as

$$
E_{u}(h)=\sqrt{\sum_{i} S_{i}\left(u_{h, i}-u_{i}\right)^{2}}, \quad E_{q}(h)=\sqrt{\sum_{j} Q_{j}\left(q_{h, j}-q_{j}\right)^{2} / \sum_{j} Q_{j}},
$$

where $S_{i}$ is the area of mesh cell $i, Q_{j}$ is the volume associated with edge $j$ (equal to the sum of the areas of the two cells separated by the edge $j$ ). Further, $q=-\boldsymbol{K} \nabla u \cdot \boldsymbol{n}$ is the edge normal flux density. Subscript $h$ refers to the discrete solution. The analytical solution $u_{i}$ is computed at the center of the cell $i$, whereas the analytical flux $q_{j}$ of cell edge $j$ is evaluated by the mid-point rule.

Table 3.1. The notations for the various schemes used in the simulations.

\begin{tabular}{ll}
\hline Notation & Algorithm Description \\
\hline LPEW1 & Linearity preserving cell-centered scheme with weights (2.26) \\
LPEW2 & Linearity preserving cell-centered scheme with weights (2.27) \\
MPFA & MPFA O-method suggested in [1] and analyzed in [14] \\
GMPFA & The generalized MPFA scheme in [26] with $\tau=0.4$ \\
MFEM & The lowest-order Raviart-Thomas mixed finite element method[12] \\
\hline
\end{tabular}

The rate of convergence can be obtained by a least squares fit on the ones computed on each two successive meshes by the following formula

$$
R_{\alpha}=\frac{\log \left[E_{\alpha}\left(h_{2}\right) / E_{\alpha}\left(h_{1}\right)\right]}{\log \left(h_{2} / h_{1}\right)}, \quad \alpha=u, q
$$

where $h_{1}, h_{2}$ denote the mesh sizes of the two successive meshes.

In addition, we denote by umin (resp., umax) the minimum (resp., maximum) value of the approximate solution.

3.1. Test 1: mild anisotropy. We consider the problem (1.1a) with full Dirichlet boundary condition (1.1b) and $\Omega=[0,1]^{2}$. A homogeneous anisotropic tensor and the exact solution are given below:

$$
\boldsymbol{K}=\left(\begin{array}{ll}
1.5 & 0.5 \\
0.5 & 1.5
\end{array}\right), \quad u(x, y)=\frac{1}{2}\left[\frac{\sin ((1-x)(1-y))}{\sin (1)}+(1-x)^{3}(1-y)^{2}\right]
$$

where the exact solution is located in the interval $[0,1]$. This test can be found in [9] as a benchmark with a slight modification for the exact solution.

We use the strictly acute triangular mesh (Mesh1), two distorted quadrilateral meshes (Mesh2 and Mesh3), randomly perturbed quadrilateral mesh (Mesh4), a locally refined nonconforming rectangular mesh (Mesh5) and a nonmatching random mesh (Mesh6) in this test (see Fig. 3.1), and the mesh refinement levels used for $L^{2}$ norm computation are also given in Fig. 3.1.

Firstly, in Table 3.2, we give the comparison of our linearity preserving cellcentered schemes LPEW1 and LPEW2 with schemes MPFA and GMPFA on the first four meshes Mesh1-Mesh4. Table 3.2 shows the following: 


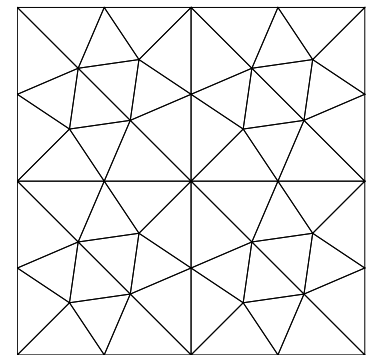

(a) Mesh1: acute triangular mesh $\left(2.5 \times 10^{-1}, 1.56 \times 10^{-2}\right)$

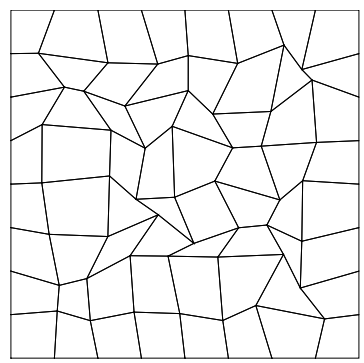

(d) Mesh4: random mesh $\left(2.85 \times 10^{-1}, 1.91 \times 10^{-2}\right)$

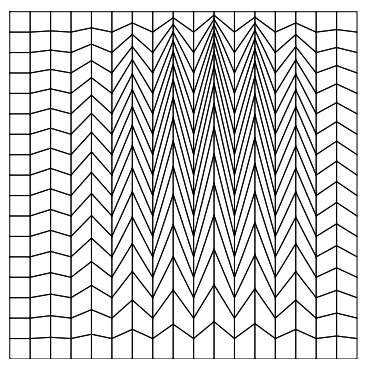

(b) Mesh2: quadrilateral mesh $\left(3.29 \times 10^{-1}, 6.72 \times\right.$ $\left.10^{-2}\right)$

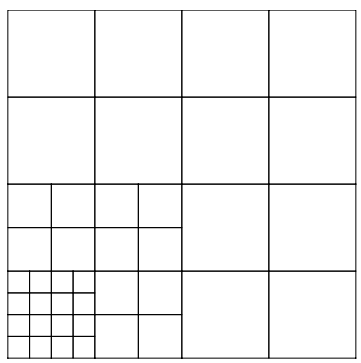

(e) Mesh5: locally refined mesh $\left(3.54 \times 10^{-1}, 2.21 \times\right.$

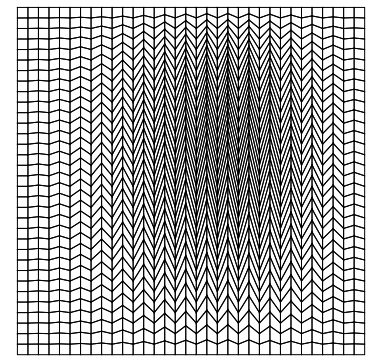

(c) Mesh3: quadrilateral mesh $\left(1.70 \times 10^{-1}, 4.27 \times\right.$ $\left.10^{-2}\right)$

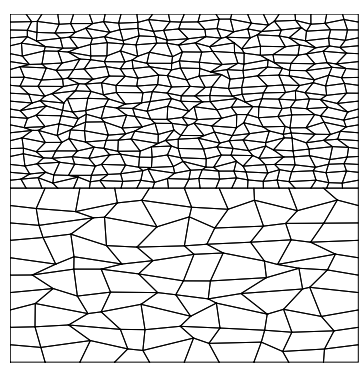

(f) Mesh6: nonmatching random mesh $\left(7.60 \times 10^{-1}, 4.56 \times\right.$ $\left.10^{-2}\right)$

Fig. 3.1. Samples of the meshes used for simulations: each mesh was used with 5 successive mesh levels, and the range of associated characteristic mesh size $h_{i}(i=1, \cdots, 5)$ is shown in the bracket as $\left(h_{1}, h_{5}\right)$ in the caption of each mesh.

(i) The minimum and maximum solutions are obtained on the coarse meshes which are exactly those in Fig. 3.1(a)-Fig. 3.1(d), and we can see that the four schemes satisfy the discrete maximum principle in this test.

(ii) All schemes show a second order convergence rate with respect to the $L^{2}$ norm of the solution on the four types of meshes except that the convergence rate of GMPFA is about $h^{1.4}$ on Mesh4.

(iii) All schemes have more than first order convergence rate with respect to the $L^{2}$ norm of the edge normal flux except schemes MPFA and GMPFA on Mesh4.

Secondly, we employ two types of nonmatching meshes (Mesh5 and Mesh6) to see how the proposed schemes LPEW1 and LPEW2 behave. The convergence rates for the solution and edge normal flux errors are graphically depicted in Fig. 3.2 as log-log plots of the discrete $L^{2}$ norm errors versus mesh size $h$. On Mesh5, the convergence rates of LPEW1 and LPEW2 for the solution and edge normal flux errors are about $h^{2}$ and $h^{1.5}$ respectively, and the convergence rates of LPEW1 and LPEW2 are around $h^{2}$ for the solution errors and $h^{1.3}$ for edge normal flux errors on Mesh6.

Finally, all the simulations demonstrate that LPEW1 and LPEW2 have desirable convergence for the solution and flux errors on triangular or distorted quadrilateral (possibly nonconforming) meshes.

3.2. Test 2: discontinuous anisotropy. In this section, we investigate the convergence of our method for a problem with a discontinuous diffusion tensor. We 
Table 3.2. minimum and maximum solutions and the convergence rates for the solution and edge normal flux errors on various meshes.

\begin{tabular}{cccccc}
\hline Mesh & Scheme & umin & umax & $R_{u}$ & $R_{q}$ \\
\hline \multirow{5}{*}{ Mesh1 } & LPEW1 & $4.02 \times 10^{-3}$ & $7.62 \times 10^{-1}$ & 1.980 & 1.665 \\
& LPEW2 & $3.99 \times 10^{-3}$ & $7.62 \times 10^{-1}$ & 1.980 & 1.711 \\
& MPFA & $2.81 \times 10^{-3}$ & $7.51 \times 10^{-1}$ & 2.000 & 1.000 \\
& GMPFA & $3.29 \times 10^{-3}$ & $7.57 \times 10^{-1}$ & 1.986 & 1.001 \\
\hline \multirow{5}{*}{ Mesh2 } & LPEW1 & $4.74 \times 10^{-4}$ & $9.11 \times 10^{-1}$ & 2.320 & 1.745 \\
& LPEW2 & $4.80 \times 10^{-4}$ & $9.11 \times 10^{-1}$ & 1.887 & 1.530 \\
& MPFA & $3.15 \times 10^{-4}$ & $9.10 \times 10^{-1}$ & 2.011 & 1.952 \\
& GMPFA & $3.14 \times 10^{-4}$ & $9.10 \times 10^{-1}$ & 2.012 & 1.894 \\
\hline \multirow{5}{*}{ Mesh3 } & LPEW1 & $8.27 \times 10^{-5}$ & $9.53 \times 10^{-1}$ & 1.573 & 1.260 \\
& LPEW2 & $1.32 \times 10^{-4}$ & $9.53 \times 10^{-1}$ & 1.933 & 1.268 \\
& MPFA & $8.88 \times 10^{-5}$ & $9.53 \times 10^{-1}$ & 1.992 & 1.952 \\
& GMPFA & $8.86 \times 10^{-5}$ & $9.53 \times 10^{-1}$ & 2.006 & 1.777 \\
\hline \multirow{5}{*}{ Mesh4 } & LPEW1 & $2.71 \times 10^{-3}$ & $8.14 \times 10^{-1}$ & 1.992 & 1.211 \\
& LPEW2 & $2.63 \times 10^{-3}$ & $8.14 \times 10^{-1}$ & 2.005 & 1.179 \\
& MPFA & $2.19 \times 10^{-3}$ & $8.07 \times 10^{-1}$ & 1.977 & 0.646 \\
& GMPFA & $2.26 \times 10^{-3}$ & $8.09 \times 10^{-1}$ & 1.413 & 0.190 \\
\hline
\end{tabular}

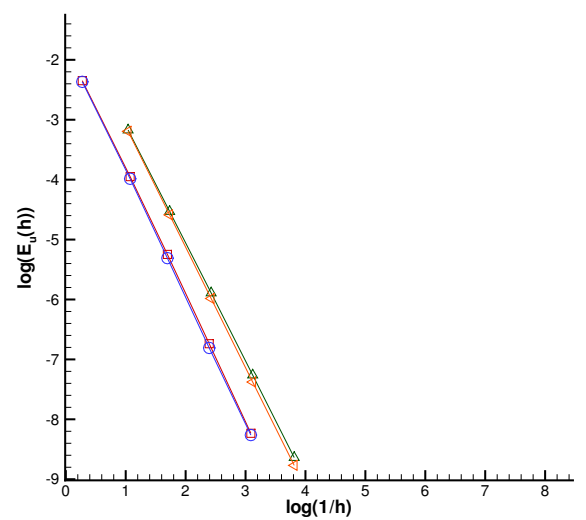

(a) solution

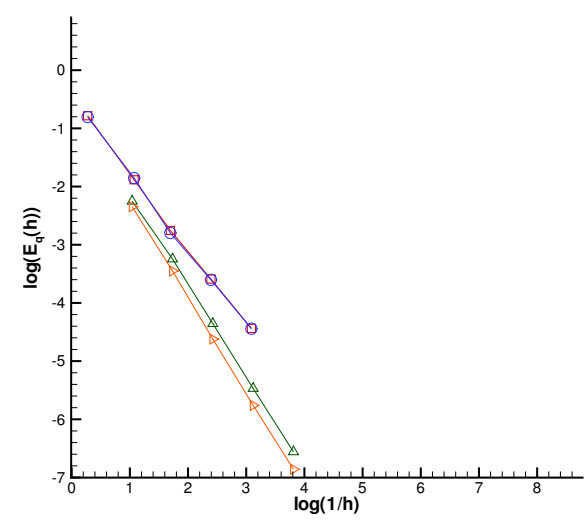

(b) edge normal flux

Fig. 3.2. Test 1: $L^{2}$ errors versus mesh size $h$ for LPEW1 and LPEW2 with symbols: $\Delta$ (LPEW1 on Mesh5), $\triangleright$ (LPEW2 on Mesh5), $\square($ LPEW1 on Mesh6), $\bigcirc($ LPEW2 on Mesh6).

solve the problem (1.1a) with full Dirichlet boundary condition (1.1b) and $\Omega=[0,1]^{2}$. Let $\boldsymbol{K}$ change the eigenvalues and orientation of eigenvectors across the line $x=0.5$,

$$
\boldsymbol{K}=\left(\begin{array}{cc}
10 & 2 \\
2 & 5
\end{array}\right), x \leq 0.5 ; \quad \boldsymbol{K}=\left(\begin{array}{cc}
1 & 0 \\
0 & 1
\end{array}\right), x>0.5
$$

We choose the following exact solution

$$
u(x, y)= \begin{cases}{[1+(x-0.5)(0.1+8 \pi(y-0.5))] \exp \left(-20 \pi(y-0.5)^{2}\right),} & x \leq 0.5 \\ \exp (x-0.5) \exp \left(-20 \pi(y-0.5)^{2}\right), & x>0.5\end{cases}
$$

This test is rebuilt from an example in [27]. The convergence behaviors of the four schemes (LPEW1, LPEW2, MPFA and GMPFA) are given on the two mesh families: the triangular mesh (Mesh1) and random mesh (Mesh4). 
To begin with, the convergence results on Mesh1 are displayed in Fig. 3.3 where we notice that the convergence rates of LPEW1 and LPEW2 for the edge normal flux errors are a little better than that of schemes MPFA and GMPFA.

Secondly in Fig. 3.4, we plot the convergence results on Mesh4. We notice that LPEW1 and LPEW2 perform very well on the discontinuous case and have a comparable convergence results with schemes MPFA and GMPFA.

Finally we ascertain that the discontinuity of the diffusion tensor does not affect the convergence rate for all the considered meshes in this test.

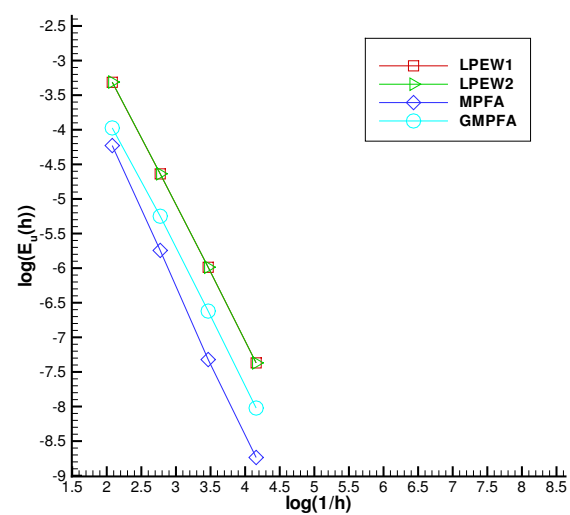

(a) solution

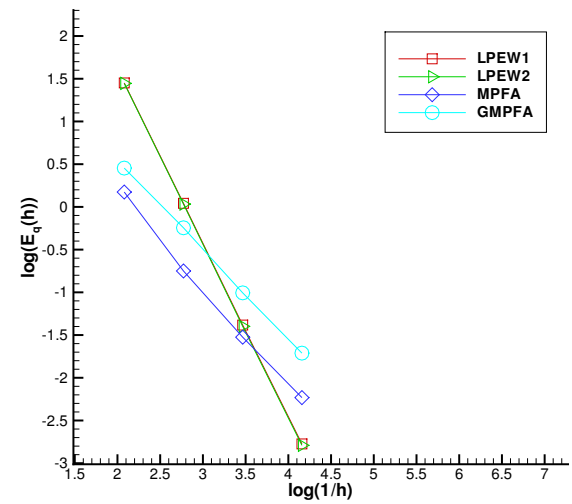

(b) edge normal flux

Fig. 3.3. Test 2: $L^{2}$ errors versus mesh size $h$ on the triangular mesh (Mesh1) .

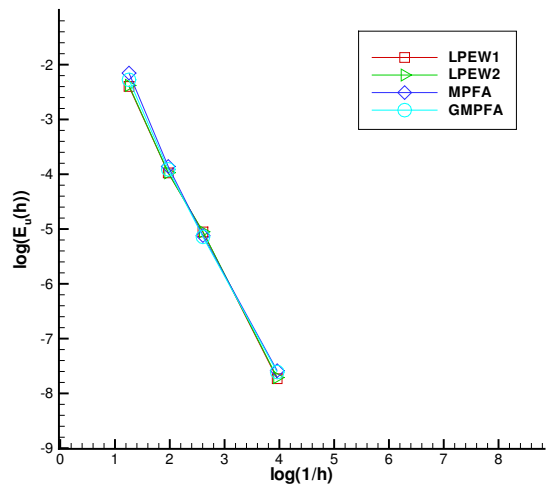

(a) solution

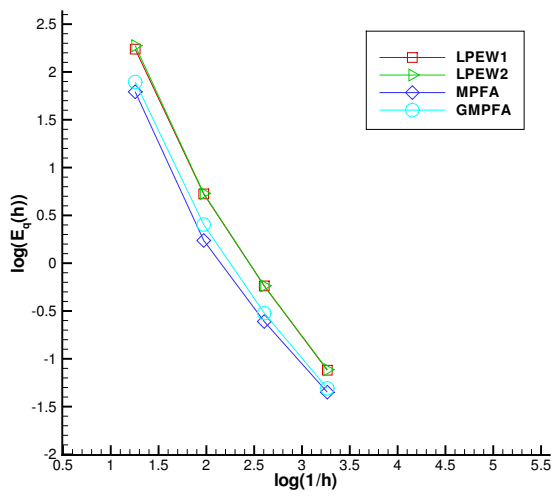

(b) edge normal flux

Fig. 3.4. Test 2: $L^{2}$ errors versus mesh size $h$ on the random mesh (Mesh4).

3.3. Test 3: heterogeneous rotating anisotropy. Problem (1.1a) with full Dirichlet boundary condition $(1.1 \mathrm{~b})$ is defined on $\Omega=[0,1]^{2}$ with the anisotropic and heterogeneous diffusion tensor field. The tensor coefficient and the exact solution are chosen to be

$$
\boldsymbol{K}=\left(\begin{array}{cc}
\alpha x^{2}+y^{2} & (\alpha-1) x y \\
(\alpha-1) x y & x^{2}+\alpha y^{2}
\end{array}\right), \quad u(x, y)=\exp \left[-20 \pi\left((x-0.5)^{2}+(y-0.5)^{2}\right)\right] .
$$


We set $\alpha>1$. The eigenvalues of $\boldsymbol{K}$ are $\lambda_{1}(x, y)=x^{2}+y^{2}$ and $\lambda_{2}(x, y)=\alpha\left(x^{2}+y^{2}\right)$. The anisotropy ratio is therefore $\alpha$ in the whole domain, and we have $\inf _{(x, y) \in \Omega} \lambda_{2}=0$ and $\sup _{(x, y) \in \Omega} \lambda_{2}=2 \alpha$ which corresponds to a highly heterogeneous case.

The sink source term $f$ corresponds to an injection at the center of the domain located between two sinks, and the magnitude of the sink source term increases as $\alpha$ increases (see Fig. 3.5). This test is inspired from [27] and induces numerical locking for some discretization schemes.

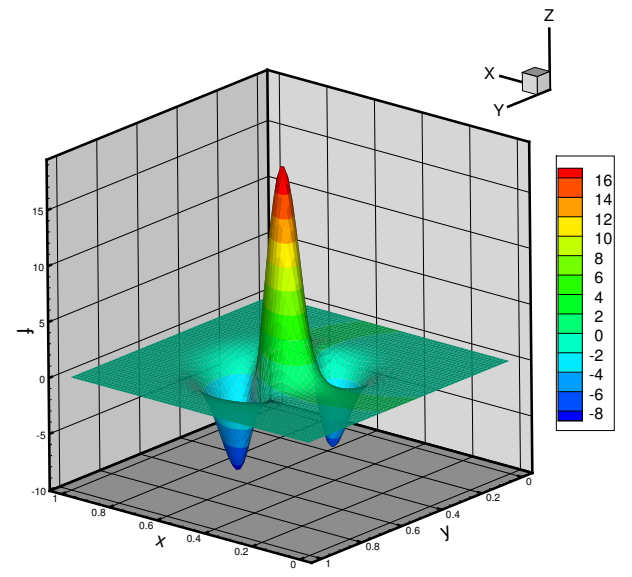

Fig. 3.5. Test 3: The distribution of the sink source term $f$ for $\alpha=10^{3}$.

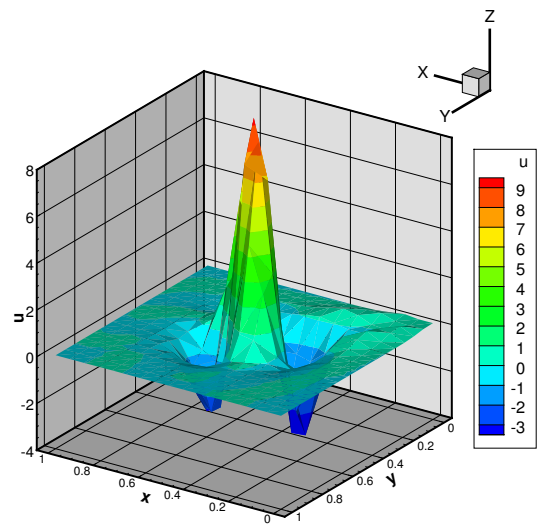

(a) MFEM

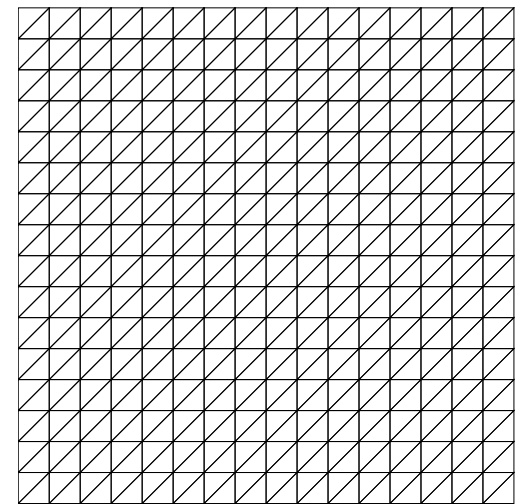

Fig. 3.6. Mesh7: uniform triangular mesh (3 meshes with mesh size $h_{i}=$ $\left.2^{1-i} \times 8.84 \times 10^{-2}, i=1,2,3\right)$.

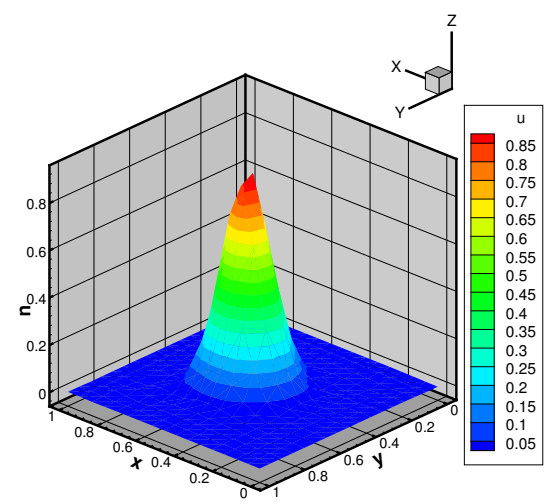

(b) LPEW2

Fig. 3.7. Test 3: The MFEM and LPEW2 solution for $\alpha=1000$ on the coarse mesh.

We study the behavior of the four schemes LPEW1, LPEW2, MPFA and MFEM for various anisotropy ratio $\alpha$ on a sequence of successively refined triangular meshes (Mesh7, see Fig. 3.6). Table 3.3 reports the minimum and maximum solutions, the solution errors and edge normal flux errors for the anisotropy ratio $\alpha=10,100,1000$ on Mesh7. The results in this table reveal the following:

(i) MPFA shows no convergence for $\alpha=100,1000$ on the finer mesh with mesh size $h_{3}=2.21 \times 10^{-2}$, and LPEW1 shows no convergence for $\alpha=1000$ on the finer mesh. But MPFA works very well on the uniform rectangular mesh as shown in [27]. 


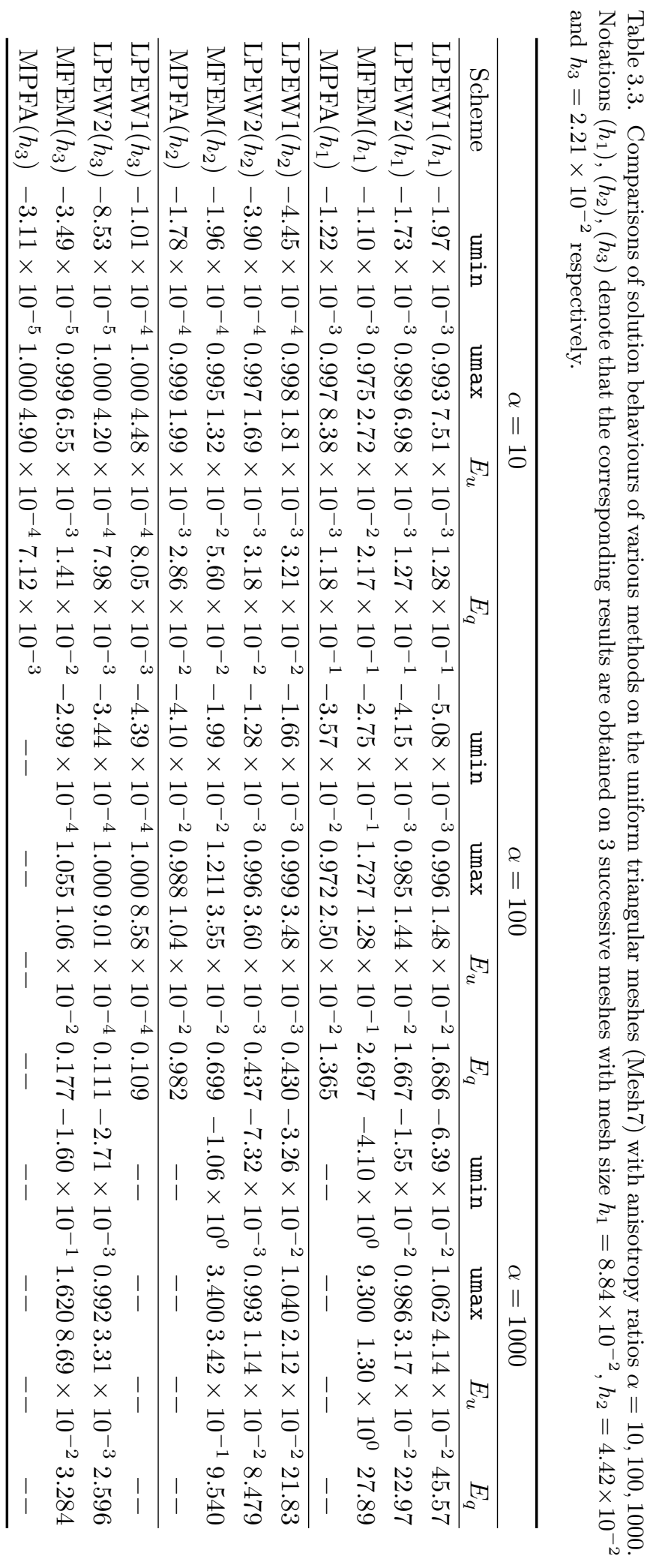


(ii) The numerical solution should be located in $[0,1]$. For anisotropy $\alpha=1000$, MFEM gives a solution with significant oscillations (umin $=-4.10 \times 10^{0}$, umax $=$ 9.300) on the coarse mesh (see Fig. 3.7(a)). These oscillations can be reduced on the finer mesh $\left(\right.$ umin $=-1.60 \times 10^{-1}$, umax $\left.=1.620\right)$.

(iii) The non-physical oscillations are avoided or strongly reduced when using our scheme. The minimum and maximum solution with LPEW2 are $-1.55 \times 10^{-2}$ and 0.986 for $\alpha=1000$ respectively on the coarse mesh, and on the finer mesh these oscillations are reduced (umin $=-2.71 \times 10^{-3}$, umax $=0.992$ ).

(iv) The solution errors with LPEW2 are much smaller than those with MFEM for various anisotropy.

(v) The convergence rates for the edge normal flux errors with MFEM and LPEW2 are very closely, and LPEW2 shows a little better than MFEM.

Finally, it should be noted that the scheme LPEW2 performs best among all the four schemes in this test and meshes, and is robust and efficient in the range of anisotropy ratio $1-1000$ which is interest in our applications.

3.4. Test 4: numerical locking. In this test, we consider the problem (1.1a) on a unit square domain $\Omega=[0,1]^{2}$ with the diffusion tensor

$$
\boldsymbol{K}=\left(\begin{array}{ll}
1 & 0 \\
0 & \delta
\end{array}\right)
$$

and the exact solution $u(x, y)=\sin (2 \pi x) e^{-2 \pi y \sqrt{1 / \delta}}$ which is dependent on $\delta$ in the $y$-direction so that the force term equals to zero. Note that the minimum and maximum values of the solution are located on the boundary, and thus are difficult to obtain with the Neumann boundary conditions. We consider the following settings for the definitions of $\Gamma_{D}$ and $\Gamma_{N}$ :

(i) Case A: fully Dirichlet boundary condition $\left(\Gamma_{N}=\emptyset\right)$;

(ii) Case B: mixed boundary condition

$$
\Gamma_{D}=\{(x, y) \mid x=0 \text { or } y=0\}, \quad \Gamma_{N}=\partial \Omega \backslash \Gamma_{D} ;
$$

(iii) Case C: nearly pure Neumann boundary condition

$$
\Gamma_{D}=\{(x, y) \mid 1-h \leq x \leq 1 \text { or } 1-h \leq y \leq 1\}, \quad \Gamma_{N}=\partial \Omega \backslash \Gamma_{D} .
$$

Case C can be treated as a modification of the fully Neumann boundary condition. The Dirichlet boundary condition is imposed on two boundary edges and the length of them tends to zero as the mesh size $h \rightarrow 0$.

To our knowledge, a locking effect $[10,18]$ may arise for strong anisotropy when solving the anisotropic diffusion model and can be experimentally observed when the discretization error does not decrease at the expected rate for the limiting anisotropic values. Test 4 was introduced in $[3,18]$ and was also used as a benchmark in the FVCA5 conference [9]. In fact some schemes could not give the solution. Severe locking effects have been reported especially in Case C $[3,11,10]$.

The meshes we consider here are the strictly acute triangular meshes (Mesh1), and the performance of LPEW2 will be investigated by calculating the solution errors and edge normal flux errors on a sequence of successively refined triangular meshes for increasing anisotropy in Case A-Case C. The performance of LPEW1 in this test and meshes is not well, so we do not display the results for LPEW1 here. 


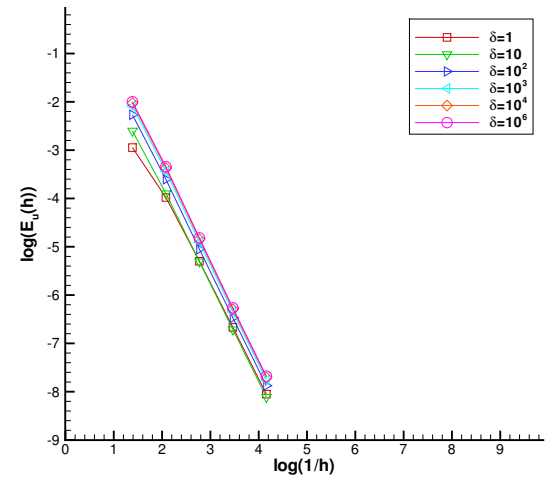

(a) Case A: solution

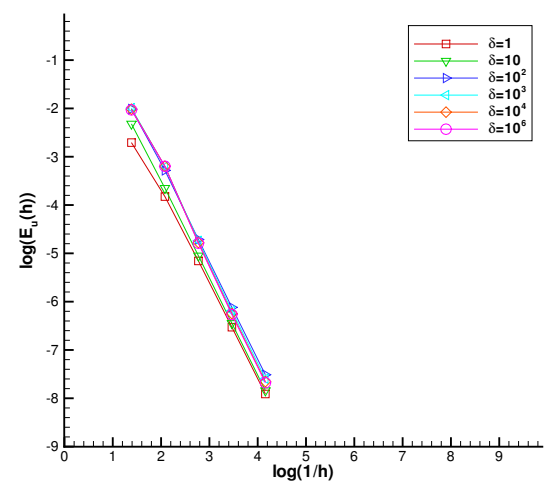

(c) Case B: solution

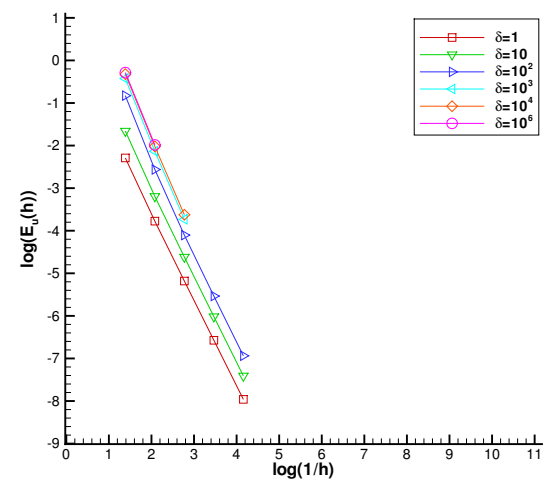

(e) Case C: solution

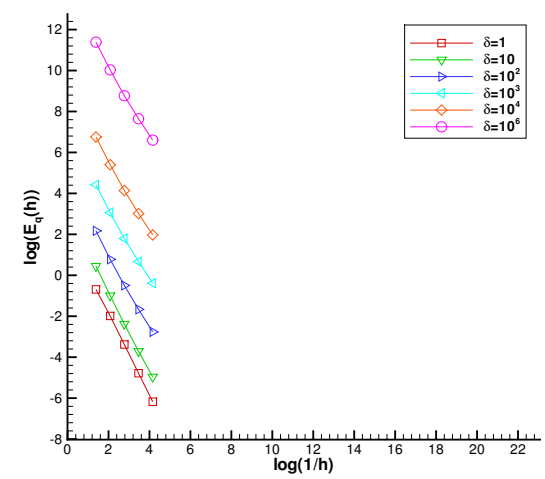

(b) Case A: edge normal flux

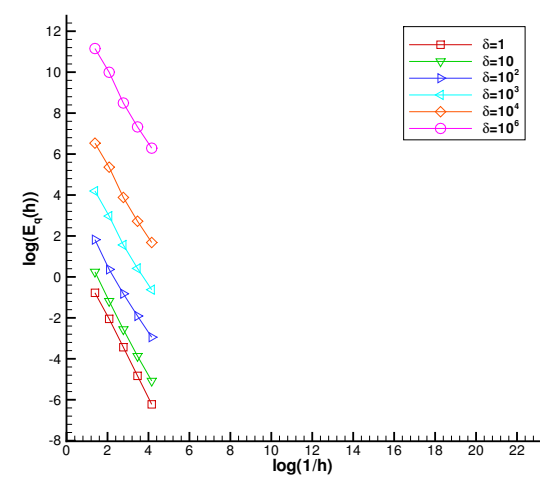

(d) Case B: edge normal flux

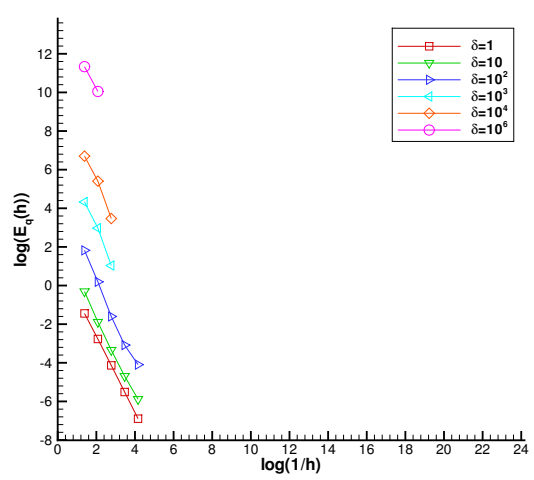

(f) Case C: edge normal flux

Fig. 3.8. Test 4: $L_{2}$ error curves for the scheme LPEW2 on the triangular mesh (Mesh1).

Table 3.4. Test 4: minimum and maximum solutions for LPEW2 on Mesh1.

\begin{tabular}{ccccccccc}
\hline \multirow{2}{*}{ Problem } & \multicolumn{2}{c}{$\delta=1$} & \multicolumn{2}{c}{$\delta=10^{2}$} & \multicolumn{2}{c}{$\delta=10^{4}$} & \multicolumn{2}{c}{$\delta=10^{6}$} \\
\cline { 2 - 9 } & umin & umax & umin & umax & umin & umax & umin & umax \\
\hline Case A & -0.716 & 0.716 & -0.897 & 0.897 & -0.931 & 0.931 & -0.935 & 0.935 \\
Case B & -0.714 & 0.717 & -0.895 & 0.895 & -0.929 & 0.930 & -0.933 & 0.934 \\
Case C & -0.838 & 0.838 & -0.858 & 0.993 & -0.773 & 0.992 & -0.769 & 0.995 \\
\hline
\end{tabular}


In Table 3.4, we present the minimum and maximum values of the solution for various anisotropy $\delta$ on the coarse mesh with mesh size $h=0.125$. Since the exact solution is located in $[-1,1]$, almost all values satisfy the discrete maximum principle.

All the convergence behaviors for LPEW2 are plotted in Fig. 3.8 as log-log plots of the $L^{2}$ norms of the solution errors and edge normal flux errors versus the characteristic mesh size $h$, and we maintain the consistency of the plot scales so that all figures can be easily comparable. The results for LPEW2 in Fig. 3.8 show the following:

(i) LPEW2 has a second order convergence rate with respect to the solution and edge normal flux errors for Case A and Case $\mathrm{B}$, and for increasing $\delta$, the convergence curves for the solution is almost the same and the solution error is deteriorated by a factor of about 1.3 going from the isotropic to the most anisotropic case.

(ii) In Case C, the presence of nearly pure Neumann boundary conditions enhances the difficulties introduced by the strong anisotropy. A locking effect is visible in Case C, and there are two symptoms: the solution error constant increases as the anisotropy $\delta$ increases; for $\delta$ up to $10^{3}$, there involve unfeasible calculations on the fine meshes with mesh size $h \leq 3.12 \times 10^{-2}$.

Finally in this test, the scheme LPEW2 can overcome or strongly reduce the numerical locking phenomenon in many cases for the triangular meshes (Mesh1).

4. Conclusion. In this article we suggest the linearity preserving cell-centered scheme, which is robust and reliable in the solution of strongly anisotropic heterogeneous diffusion problems on general distorted (possibly nonconforming) meshes. The scheme allows arbitrary diffusion tensors, and the computation of the weights in the scheme is neither discontinuity dependent nor mesh topology dependent. Many dedicate experiments using meshes with unstructured, severely distorted and nonmatching elements and arbitrary (continuous or discontinuous, homogeneous or heterogeneous) anisotropic diffusion tensors show the good performance of the proposed scheme (quadratic convergence rate for the approximate solution and higher than first order accuracy for the discrete flux).

\section{REFERENCES}

[1] I. Aavatsmark, T. Barkve, Ø. BøE And T. Mannseth, Discretization on non-orthogonal, quadrilateral grids for inhomogeneous, anisotropic media, J. Comput. Phys. 127 (1996) $2-14$.

[2] I. Aavatsmark, G. T. Eigestad, R. A. Klausen, M. F. Wheeler, I. Yotov, Convergence of a symmetric MPFA method on quadrilateral grids, Comput. Geosci., 11 (2007) 333-345.

[3] I. BABUSKA AND M. SURI, On locking and robustness in the finite element method, SIAM J. Numer. Anal. 29 (5) (1992) 1261-1293.

[4] M. M. Basko, J. Maruhn and An. Tauschwitz, An efficient cell-centered diffusion scheme for quadrilateral grids, J. Comput. Phys. 228 (2009) 2175-2193.

[5] J. Breil And P.-H. Maire, A cell-centered diffusion scheme on two-dimensional unstructured meshes, J. Comput. Phys. 224 (2007) 785-823.

[6] L. N. Chang and G. W. Yuan, Cell-centered finite volume methods with flexible stencils for diffusion equations on general nonconforming meshes, Comput. Methods Appl. Mech. Engrg. 198 (2009) 1638-1646.

[7] Q. -Y. Chen, J. Wan, Y. YAng And R. T. Mifflin, Enriched multi-point flux approximation for general grids, J. Comput. Phys. 227 (2008) 1701-1721.

[8] Y. Coudire, J.-P. Vila ANd P. Villedieu, Convergence rate of a finite volume scheme for a two-dimensional diffusion convection problem, Math. Model. Numer. Anal. 33 (3) (1999) 493-516.

[9] R. Herbin And F. Hubert, Benchmark on Discretization Schemes for Anisotropic Diffusion Problems on General Grids. In Finite Volumes for Complex Applications V - Problems \&Perspectives, Edited by: R. Eymard, J.-M. Herard. pp 659-692, Wiley press, 2008. 
[10] V. HAVu, An analysis of asymptotic consistency error in a parameter dependent model problem, Calcolo 40 (2) (2003) 121-130.

[11] V. Havu And J. PitKaranta, An analysis of finite element locking in a parameter dependent model problem, Numer. Math. 89 (4) (2001) 691-714.

[12] F. Hecht, O. Pironneau and J. Morice, Freefem ++ manual, 2008. Available from: http://www.freefem.org.

[13] W. Z. Huang and A. M. Kappen, A study of cell-center finite volume methods for diffusion equations, Mathematics Research Report 98-10-01, University of Kansas, 1998.

[14] R. A. Klausen and R. Winther, Robust convergence of multi point flux approximation on rough grids, Numer. Math. 104 (2006) 317-337.

[15] K. Lipnikov, M. Shashkov and I. Yotov, Local flux mimetic finite difference methods, Numer. Math. 112 (2009) 115-152.

[16] D. Y. Li, H. S. Shui And M. J. TAng, On the finite difference scheme of two-dimensional Parabolic equation in a Non-Rectangular Mesh, J. Numer. Methods Comput. Appl. 1(1980) 217-224.

[17] G. X. Lv, L. J. Shen And Z. J. Shen, Numerical methods for energy flux of diffusion equation on unstructured grids (in Chinese), Chinese Journal of Computational Physics, 24(4) (2007) 379-386.

[18] G. Manzini And M. Putti, Mesh locking effects in the finite volume solution of 2-D anisotropic diffusion equations, J. Comput. Phys. 220 (2) (2007) 751-771.

[19] J. M. Morel, J. E. Dendy Jr, M. L. Hall, and S. W. White, A cell-centered Lagrangianmesh diffusion differencing scheme, J. Comput. Phys. 103 (1992) 286-299.

[20] J. E. Morel, R. M. Roberts And M. J. Shashkov, A local support-operators diffusion discretization scheme for quadrilateral $r-z$ meshes, J. Comput. Phys. 144 (1998) 17-51.

[21] M. Shashkov and S. Steinberg, Solving diffusion equations with rough coefficients in rough grids, J. Comput. Phys. 129 (1996) 383-405.

[22] Z. Q. Sheng And G. W. Yuan, A nine point scheme for the approximation of diffusion operators on distorted quadrilateral meshes, SIAM J. Sci. Comput. 30 (2008) 1341-1361.

[23] M. F. Wheeler And I. Yotov, A multipoint flux mixed finite element method, SIAM J. Numer. Anal., 44 (2006) 2082-2106.

[24] J. M. Wu, S. W. Fu AND L. J. Shen, A difference scheme with high resolution for the numerical solution of a nonlinear diffusion equation, J. Numer. Methods Comput. Appl. 24 (2003) 116-128; translation in Chinese J. Numer. Math. Appl. 25 (2003) 79-94.

[25] J. M. Wu, Linearly exact method and the difference scheme for diffusion equation on quadrilateral meshes, Annual report, Laboratory of Computational Physics, Institute of Applied Physics and Computational Mathematics, Beijing, China, 2005, 156-169.

[26] J. M. WU, Z. H. DAI, Z. M. GAO AND G. W. YUAN, Linearity preserving nine-point schemes for diffusion equation on distorted quadrilateral meshes, to appear, 2009.

[27] A. Younes AND V. Fontaine, Efficiency of mixed hybrid finite element and multipoint flux approximation methods on quadrangular grids and highly anisotropic media. Int. J. Numer. Engng. 2008: 76: 314-336.

[28] Q. ZhaO And G. W. Yuan, Analysis and construction of cell-centered finite volume scheme for diffusion equations on distorted meshes, Comput. Methods Appl. Mech. Engrg. 198 (2009) 3039-3050. 